\title{
La transferencia de conocimiento científico y tecnológico: un reto que deben afrontar las universidades
}

Óscar D. Molina ${ }^{1}$

A partir de los precedentes que han marcado la evolución de las universidades, es propicio referirse al nuevo enfoque bajo el cual se empieza a entretejer de forma creciente y planificada la tercera misión universitaria (las otras dos son docencia e investigación): la cual consiste en promover que los nuevos conocimientos científicos, ideas creativas y tecnologías generadas en el seno de la universidad se pongan al servicio de la sociedad, se transformen en nuevos productos o servicios valiosos para el mercado y, por tanto, generen riqueza y bienestar social. Esta nueva dimensión se sustenta en lo que hoy se denomina como las universidades emprendedoras (Clarck, 1999), las cuales incorporan en la agenda de la investigación científica a las políticas de transferencia y explotación de conocimientos y propiedad intelectual, entre otros.

En este contexto, el nuevo enfoque de la tercera misión universitaria (antes conocida de forma genérica como extensión universitaria) gravita sobre la base de dos grandes ejes: por un lado, la responsabilidad social institucional de la universidad; y, en segundo lugar, el compromiso ineludible de transformar el conocimiento científico y tecnológico en valor económico, incidiendo en la competitividad y productividad de los países, facilitando la innovación, la creatividad y el desarrollo cultural, científico y tecnológico.

Al respecto, se están planteando iniciativas que, desde el punto de vista político, pretenden favorecer la interrelación e integración entre los procesos de I+D de las universidades para el fomento de la innovación. Estas nuevas políticas tienen particular énfasis en el establecimiento de nuevos mecanismos de transferencia de conocimiento científico.

La manera en la que el conocimiento se transfiere de las instituciones de educación superior a una sociedad, han variado dependiendo del tipo de conocimiento que es

1 Departamento de Propiedad Intelectual e Innovación, Dirección de Investigación Científica y Posgrado, UNAH: investigacionunah.pi.innov.om@gmail.com; oscarmolina.unah@yahoo.com 
transferido y de los mecanismos que se han adoptado para hacerlo. Históricamente, las publicaciones de revista científicas ha sido el medio propicio para la diseminación del conocimiento científico básico, sin embargo, el debate empieza a surgir a partir del cuestionamiento sobre el conocimiento científico que resulta de las investigaciones aplicadas, ya que estas representan un activo intangible potencial que involucra otros intereses, más allá de lo que representa la publicación y la divulgación.

No obstante, aunque la enseñanza, la publicación y la divulgación sean eficaces para transferir conocimiento, para el logro de avances en una disciplina, han sido necesarios, en la práctica, nuevos métodos para transferir el conocimiento a la sociedad. Hablamos particularmente sobre los mecanismos que vinculen la protección, explotación o comercialización del conocimiento, tales como patentes, licencias o creación de empresas de base tecnológica (spin off).

Basándose en lo anterior y atendiendo al principio de que la sociedad cada vez más demanda de las universidades el usufructo del conocimiento científico y tecnológico, el hecho de publicar en revistas los trabajos o resultados científicos no es, ni mucho menos, condición sine qua non para asegurar que los destinatarios finales (la sociedad en su conjunto) se apropien de este conocimiento para transfórmalo y convertirlo en activos intangibles. Es a partir de este momento en donde las universidades empiezan a replantear la necesidad de promover, a la par de la investigación básica o fundamental, la investigación aplicada que, por su naturaleza, es la que induce a plantear nuevos modos de transferencia del conocimiento.

En virtud de los anterior, vale la pena enfatizar que la investigación básica siempre seguirá siendo el pilar que sustenta el quehacer de las universidades en términos de producción de conocimiento, ya sea para llenar vacíos conceptuales o para seguir fortaleciendo el acervo de conocimiento de un campo en específico. No obstante, en el nuevo contexto de la tercera misión, la investigación aplicada cada vez toma más preeminencia en las universidades, pues estaconstituye el vehículo principal para hacer efectiva la responsabilidad social de contribuir en la solución de problemas.

Las universidades como instituciones fundamentales para liderar la producción de nuevo conocimiento, están llamadas a hacer un verdadero encuentro entre lo que es la investigación científica y lo que corresponde consecuentemente a las etapas del desarrollo tecnológico, la innovación y la propiedad intelectual. Para ello, además de articular sus funciones sociales, deberá contextualizar la ciencia y la tecnología en escenarios de aplicación, superar las anacrónicas e históricas divisiones entre las 
disciplinas y, sobre todo, crear sinergias a partir de lo que constituye el modelo de la cuádruple hélice (vinculación de las universidades con el gobierno, el tejido productivo y la sociedad), desarrollando colaborativa y coparticipativamente investigaciones con los diferentes actores, coadyuvando a la solución de problemas como fin.

Así, el impulso a los procesos de transferencia de conocimiento científico se ha planteado como una de las principales vías de acceso para fomentar una cultura de investigación que propenda, a la luz de un enfoque epistemológico y ontológico, descifrar las realidades sociales para interpretar sus propias causalidades y consecuentes necesidades, para plantear de manera pertinente investigaciones que prescriban un alcance cuyo fin de aplicación persigan el desarrollo tecnológico y la innovación.

La transferencia del conocimiento se ha convertido, de este modo, en la tercera misión de la universidad, que se une a las dos misiones históricas, docencia e investigación. En su esencia, la tercera misión de la universidad es el término que se emplea actualmente para definir las actividades ligadas a la generación, aplicación, uso y explotación del conocimiento, la tecnología y la innovación que se genera dentro de los propios límites de la docencia y la investigación.

Es en este escenario que ha proliferado la creación de instrumentos facilitadores para el desarrollo de la actividad de I+D+i en las universidades. Por ejemplo, la instauración de unidades de creación de empresas (spin-off), la creación de oficinas de transferencia tecnológica (OTT) como vía expedita del traslado de resultados y otras medidas similares que ilustran la voluntad de la universidad en esta materia, con respecto a la explotación de la investigación tecnológica, el desarrollo tecnológico, la innovación y la propiedad intelectual.

La creación de empresas, comúnmente llamadas "spin-off", presenta ciertas ventajas con respecto a las otras vías de transferencia. En primer lugar, constituye la vía más directa para la comercialización de la tecnología y, en segundo lugar, supone una alternativa para la explotación de resultados cuando la naturaleza de la nueva tecnología hace de las patentes un método de protección poco efectivo o no permite a los investigadores capturar todo el valor mediante los acuerdos de licencia.

Lo anterior apunta a que las universidades están inmersas en la dinámica de apoyo a la actividad de $\mathrm{I}+\mathrm{D}+\mathrm{i}$, ya que se encaminan hacia modelos de gestión y 
transferencia de la investigación que intermedien entre los intereses académicocientíficos y los omerciales demandados por el mercado. En consecuencia, las "spin-off" universitarias se convierten en un punto de contacto entre ambas realidades, de ahí su notoriedad como herramienta estratégica para favorecer la interoperabilidad de la I+D+i desde los propios escenarios académicos.

Las empresas de base tecnológica como un mecanismo de transferencia de los resultados de investigación, son consideradas por muchos expertos como una de las fórmulas más eficaces para valorar el conocimiento de la universidad y para afianzar las relaciones entre el tejido productivo y el ámbito científico y académico. Mediante su creación, las universidades contribuyen al desarrollo económico y social de la región a través de la creación de empleo y riqueza, del desarrollo de nuevas tecnologías y de la capacidad de generar un alto valor añadido a la actividad económica, debido a que la investigación aplicada es la base de las empresas de base tecnológica universitarias, ya que generalmente utilizan el conocimiento desarrollado en sus instituciones de origen para crear productos o procesos innovadores.

De forma general, los autores especializados definen las empresas de base tecnológica universitarias como nuevas empresas creadas para comercializar una invención desarrollada por la universidad y fundadas por personal de la propia institución. Siguiendo la definición de O'Shea y otros (2008), la creación de empresas de base tecnológica debe comprender por lo menos dos características: la primera es que sea creada producto de una investigación y la segunda es que la investigación debe haber formado parte un investigador académico. Por su parte, la Office of Technology Assessment (1992) las define como organizaciones productoras de bienes y servicios, comprometidas con el diseño, desarrollo y producción de nuevos productos o procesos de fabricación innovadores, mediante la aplicación sistemática de conocimientos técnicos y científicos.

Por todas estas razones, la transferencia de conocimiento y su labor en el fomento del emprendimiento sobre la base de la I+D, adquiere más importancia que nunca en un sistema en el que se requiere que la universidad traspase sus muros y revierta en la sociedad la generación del conocimiento científico e innovador en forma de investigación aplicada y en la creación de empresas de base tecnológica.

En tal sentido, las relaciones entre las universidades, el gobierno y el tejido empresarial son claves para el cambio de modelo productivo que necesita nuestra sociedad. 
Del mismo modo, el emprendimiento ha dejado de ser una oportunidad para convertirse en una necesidad y las universidades adquieren un papel relevante en la tarea de su sensibilización y promoción.

Como puede observarse, la tercera misión de las universidades agrupa tareas difíciles de cuantificar y dimensionar, pero inexorablemente se avanza en la concreción de este nuevo enfoque universitario, lo que permite poner de relieve la importancia de diseñar estrategias y políticas orientadas a estos nuevos estamentos para favorecer la generación, el intercambio, la transferencia, la comercialización y la explotación del conocimiento científico y tecnológico. Avanzar en la construcción de estos andamiajes permitirá a las universidades reducir progresivamente la brecha que suponen esos nuevos estadios asociados a las actividades básicas de la universidad.

No obstante, aunque los avances de nuestras universidades en estas nuevas dimensiones son muy incipientes, diversos análisis detectan que mientras las universidades se apropien y se aproximen a estos escenarios de ciencia y tecnología, deberán ir propiciando ciertas acciones conducentes a estos objetivos:

1. Sensibilizar a la comunidad académica y aumentar el conocimiento sobre los beneficios potenciales de la investigación aplicada con orientación a la solución de problemas.

2. Crear sinergias con empresas que valoren la $I+D$ de las universidades y que puedan tener intereses específicos en áreas de conocimiento particulares.

3. Trabajar en colaboración con ámbitos de conocimiento que, a priori, pueden tener una mayor ventaja competitiva en la generación de conocimiento científicotecnológico.

4. Promover la participación de investigadores, profesores, la misma universidad, estudiantes de grado y posgrado y aliados estratégicos privados y públicos, para desarrollar incubadoras de empresas, empresas de base tecnológica y otras formas novedosas de transferencia de conocimiento en el marco de la cuádruple hélice.

5. Identificar nuevos retos de investigación relevantes para los diferentes sectores (social y productivo). 
Para las universidades de Honduras, la tercera misión representa un fenómeno relativamente reciente, tanto en el ámbito de estudio como en la realidad práctica. Sin embargo, instituciones como la UNAH han empezado a valorar esta propuesta a partir de la construcción de la cultura de I+D+i, la cual ha sido liderada y, a su vez catalizada, por la Dirección de Investigación Científica y Posgrado (DICYP). En virtud de ello, la DICYP sobre la base de sus preceptos, ha empezado a rebatir el paradigma tradicional de la investigación con una visión más holística y sistémica que propende, basándose en el enfoque de la investigación tecnológica, a estimular y potenciar el recurso humano, las capacidades y habilidades de creación, invención y generación de conocimientos científicos y tecnológico. Estas acciones buscan fortalecer las actitudes proactivas y creativas de los actores universitarios para transformar el conocimiento tácito en conocimiento descodificado, a través de figuras concretas como la innovación y la propiedad intelectual, a luz de la propia interpretación de los problemas de la sociedad.

En este sentido, la DICYP ha empezado a poner en el escenario de sus procesos de gestión y dirección, mecanismos dinamizadores que buscan encausar una relación simbiótica entre lo que hacen las unidades académicas, en lo que corresponde a las actividades de investigación, y lo que se plantea la política institucional de I+D+i como objetivo. De esta forma, los avances van focalizados a diseñar y liderar planes estratégicos de organización gestión, ejecución, transferencia, explotación y comercialización de activos intangibles bajo expresiones genuinas de productos, procesos o servicios de valor agregado.

Para lograr dichos propósitos, la DICYP ha diseñado diversas estrategias que implican una nueva cosmovisión basada en la cultura de emprendimiento de la I+D, la cual mediante acciones sucesivas aspira a llegar a escenarios de ciencia y tecnología, tales como las incubadoras de empresas y las empresas de base tecnológica universitarias, favoreciendo la interconexión con el tejido social, económico y productivo en el desarrollo de proyectos colaborativos o coparticipativos, con valor agregado y con orientaciones a la solución de problemas en el plano local, regional y nacional.

En resumen, la UNAH observa con optimismo un clima organizacional más dinámico y flexible que cada vez es más proclive a asimilar con visión de futuro estos escenarios de ciencia y tecnología. 


\section{Referencias}

Clark, B. R. (1999). Creating Entrepreneurial Universities: Organizational. Pathways of transformation. Higher Education, 38(3), 373-377.

Office Of Technology Assesstment. (1992). Building Future Security: Strategies for Restructuring the Defense Technology and Industrial Base. Princeton: OTA.

O'Shea, R. P.; Chugh, H. \& Allen, T. J. (2008). Determinants and consequences of university spin off activity: a conceptual framework. The Journal of Technology Transfer, 33(6), 653-666. 